\title{
Elucidating the molecular bases of epigenetic inheritance in non-model invertebrates: the case of the root-knot nematode Meloidogyne incognita
}

\section{Laetitia Perfus-Barbeoch ${ }^{1,2,3}$, Philippe Castagnone-Sereno ${ }^{1,2,3}$, Michael Reichelt ${ }^{4}$, Sara Fneich ${ }^{5,6}$, David Roquis $^{5,6}$, Loris Pratx ${ }^{1,2,3}$, Céline Cosseau ${ }^{5,6}$, Christoph Grunau ${ }^{5,6}$ and Pierre Abad ${ }^{1,2,3}$}

\author{
1 INRA, Institut Sophia Agrobiotech, UMR 1355 ISA, Sophia-Antipolis, France \\ ${ }^{2}$ CNRS, Institut Sophia Agrobiotech, UMR 7254 ISA, Sophia-Antipolis, France \\ ${ }^{3}$ Institut Sophia Agrobiotech, Université de Nice Sophia-Antipolis, UMR ISA, Sophia-Antipolis, France \\ ${ }^{4}$ Abteilung Biochemie, MPI für Chemische Ökologie, Jena, Germany \\ ${ }^{5}$ Ecologie et Evolution des Interactions, Université de Perpignan Via Domitia, Perpignan, France \\ ${ }^{6}$ Ecologie et Evolution des Interactions, CNRS, UMR5244, Perpignan, France
}

\section{Edited by:}

Mackenzie Gavery, University of Washington, USA

Reviewed by:

Guilherme Corrêa De Oliveira,

Fundação Oswaldo

Cruz - FIOCRUZ-Minas, Brazil

Elena Gómez-Díaz, Emory

University, USA

*Correspondence:

Laetitia Perfus-Barbeoch, INRA, UMR 1355 ISA, CNRS, UMR 7254

ISA, Institut Sophia Agrobiotech,

Université de Nice Sophia-Antipolis,

400 route des Chappes, BP167,

Sophia Antipolis cedex,

Alpes-Maritimes, France

e-mail: zurletto@sophia.inra.fr
Root-knot nematodes of the genus Meloidogyne are biotrophic plant parasites that exhibit different life cycles and reproduction modes, ranging from classical amphimixis to obligatory mitotic parthenogenesis (apomixis), depending on the species. Meloidogyne incognita, an apomictic species, exhibits a worldwide distribution and a wide host range affecting more than 3000 plant species. Furthermore, evidences suggest that apomixis does not prevent $M$. incognita from adapting to its environment in contrast to what is expected from mitotic parthenogenesis that should theoretically produce clonal progenies. This raises questions about mechanisms of genome plasticity leading to genetic variation and adaptive evolution in apomictic animals. We reasoned that epigenetic mechanisms might in part be responsible for the generation of phenotypic variants that provide potential for rapid adaptation. We established therefore a pipeline to investigate the principal carriers of epigenetic information, DNA methylation and post-translational histone modifications. Even if $M$. incognita possesses the epigenetic machinery i.e., chromatin modifying enzymes, 5-methyl-cytosine and 5-hydroxy-methyl-cytosine content is absent or very weak. In contrast, we demonstrated that the canonical histone modifications are present and chromatin shows typical nucleosome structure. This work is the first characterization of carriers of epigenetic information in $M$. incognita and constitutes a preamble to further investigate if $M$. incognita development and its adaptation to plant hosts are under epigenetic control. Our pipeline should allow performing similar types of studies in any non-model organism.

Keywords: epigenetics, Meloidogyne incognita, chromatin, DNA-methylation, histone modification

\section{INTRODUCTION}

Epigenetics is the study of heritable changes in gene expression and function that cannot be explained by changes in DNA sequence. These molecular mechanisms can be influenced by the environment and the individual history as well as being potentially transferable from a generation to the next, with a reversible character (Youngson and Whitelaw, 2008). Organisms are forced to adapt to the heterogeneousness of the environment and the phenotypic plasticity constitutes a major adaptation to compulsory constraints. Since the system of reproduction of an organism conditions its evolutionary dynamics as well as the demography and the spatial genetic structuring of its populations, it is of particular relevance to study epigenetic variation in asexually reproducing organisms. Indeed, in spite of a genetic homogeneity in asexually reproducing organisms, an important phenotypic variability can be observed between individuals of the same clonal lineage found in different environments. The influence of the epigenetic mechanisms could bring an explanation to this paradox (reviewed in Verhoeven and Preite, 2014). Similarly, epigenetic modifications may provide an accessory source of fastacting, reversible, and readily available phenotypic variations that can shape host-pathogen interactions (reviewed in Gómez-Díaz et al., 2012). Despite growing evidences on the role of epigenetic phenomena on a wide range of biological phenomena, many questions remain for the involvement of epigenetics in plasticity and adaptation.

Root-knot nematodes (RKN) of the genus Meloidogyne are obligatory plant parasites that constitute major agricultural pests worldwide. They infect almost all cultivated plants and establish an intimate interaction with their hosts inducing the redifferentiation of root cells into hypertrophied and multinucleate feeding cells (Caillaud et al., 2008). So far, the most efficient control measure against RKN was the use of chemical substances called nematicides. However, because of their toxic side effects on the environment, nematicides have recently been banned from use. Novel approaches to control them are sorely needed. Among 
RKN species, some are strict or facultative amphimitic while others reproduce exclusively by mitotic parthenogenesis (apomixis) (Castagnone-Sereno, 2006). Our model, Meloidogyne incognita, reproduces in an asexual way by parthenogenesis without meiosis. Genetically identical individuals develop from $M$. incognita females and form virtually clonal populations. Although these clones share the same genetic heritage, modifications of their phenotype can be observed when they are exposed to unfavorable environments (Castagnone-Sereno et al., 1994). These phenotypes can be completely different such as juveniles molting into either female or male, but also show for instance a switch of virulence leading to infection of resistant hosts. A first step toward a better understanding of plant-nematode interaction was the sequencing of the genome of $M$. incognita. Analysis of this reference genome highlighted characteristics, which could be at the origin of the parasitic success of this species (Abad et al., 2008; Danchin et al., 2010).

Epigenetics changes refer to a set of molecular processes that can affect gene expression such as regulatory processes mediated by remodeling of chromatin structure through methylation of cytosine residues in the DNA and chemical modifications of histone proteins, in particular their acetylation or methylation (Jablonka and Lamb, 2002). On one hand, DNA methylation refers to a chemical modification of genomic DNA by the addition of a methyl (-CH3) group to specific nucleotide bases. The most common form of DNA methylation is cytosine methylation, occurring predominantly in CpGs in animal genomes (Suzuki and Bird, 2008). However, because of lack of de novo methylation machinery, DNA methylation process is unlikely to occur in nematodes, including $C$. elegans but with the exception of Trichinella spiralis (Simpson et al., 1986; Wenzel et al., 2011; Gao et al., 2012, 2014). On the other hand, post-translational modifications of histone amino acid side chains (PTMs) are known to mediate epigenetic regulation of gene expression and development in animals (Li et al., 2007; Luger et al., 2012; Turner, 2012). For instance, PTMs, such as acetylation of histone $\mathrm{H} 3$ at lysine 9, are typically found in euchromatin and often associated with active transcription, whereas other modifications, such as methylation of lysine 9 or lysine 27 of histone H3, are found in heterochromatin and related to gene repression (Jenuwein and Allis, 2001). One major role for epigenetic variation in evolution would be to promote phenotypic variability, and allow populations to widely explore new environmental conditions. This could lead to a rapid adaptation to environmental changes or colonization of new environments. The interactions between parasites and their host are models of choice to study these mechanisms because the selective pressures are strong and the evolution is fast. M. incognita constitutes an ideal model to study these mechanisms, especially as clones can be obtained following the naturally occurring asexual multiplication in host plants. Whereas variation at the genetic level has been studied in M. incognita, less is known about the extent and function of epigenetic variation. Previous analyses on multiple $M$. incognita populations have shown that genomic polymorphism could not be directly correlated to observed phenotypes (Semblat et al., 2000).

Many non-model organisms of ecological and economical importance such as M. incognita suffer from a lack of even rudimentary knowledge about their epigenetic information carriers. To address this problem, we describe here a straightforward $2 \times 3$-step procedure to perform a fundamental analysis of elements of the chromatin marking system. We first performed a whole genome bioinformatics search for chromatin-modifying enzymes such as those involved in DNA methylation, we evaluate the degree of DNA methylation by calculating the $\mathrm{CpG}$ observed/expected ratio in $M$. incognita transcriptome and by measuring DNA methylation levels of 5-methylcytosine (5-mC) and 5-hydroxy methylcytosine (5-hmC) in genomic DNA by LCMS/MS. We then used Western blots to verify the presence of histone and histone modifications and tested critical parameters of a standardized N-ChIP procedure that need to be adapted for each model.

\section{ANALYSIS OF DNA METHYLATION}

DNA methylation is a common feature of many but not all genomes. We recommend starting with this feature.

\section{STEP 1: IN-SILICO SEARCHES OF CHROMATIN-REMODELING ENZYMES}

To understand whether $M$. incognita possesses the capacity to methylate DNA, we first conducted reciprocal BLASTp searches combined to OrthoMCL analysis to identify proteins that might be related to known chromatin-remodeling enzyme orthologs (Table 1). Significant hits were defined as those satisfying the following criteria: $E$-value $<10^{-5}$ and the aligned segments covering at least $30 \%$ of the sequence length of the hit. OrthoMCL has been applied to the proteome data set from $M$. incognita and three publicly available nematode genomes (C. elegans, T. spiralis, and Pristionchus pacificus). Animal DNA methyltransferases can be subdivided into three subfamilies, DNMT-1, DNMT2 and DNMT-3, based on sequence similarity (Kumar et al., 1994; Hendrich and Tweedie, 2003). DNMT-1 has been regarded as a "maintenance" methyltransferase, whereas DNMT-3 functions as "de novo" methyltransferase and some evidence suggest that DNMT-2 enzymes are active cytosine-5'-methyltransferases (Kunert et al., 2003). Species exhibiting functional DNA methylation generally encode a complete set of DNMTs in their genomes whereas species lacking DNA methylation, such as C. elegans, have lost DNA methylation enzymes from their genomes (Gutierrez and Sommer, 2004; Suzuki and Bird, 2008). BLASTp search of the $M$. incognita whole proteome (Supplementary Material) was performed with DNMT-1, DNMT-2, DNMT-3 protein sequences from nematodes (Table 1): the Trichinella spiralis DNMT-1 (accession number EFV58204.1); P. pacificus Ppa- DNMT-2 (accession number AY766101.1) and T. spiralis DNMT-22 (accession number EFV60295.1); T. spiralis DNMT-3 (accession number EFV54759.1). As for the 11 other nematodes in which DNA methylation has been studied, with exception of T. spiralis (Gao et al., 2012), M. incognita did not possess de novo methylation machinery, DNMT-3 nor DNMT-2. However, our data revealed the existence of a potential DNMT-1 orthologous protein annotated in $M$. incognita, as well as methyl-CpG binding domain (MBDs) proteins, which encodes another essential component of the methylation system (Table 1). We found that Minc01117 could be ortholog to the maintenance methyltransferase DNMT1 from other species. Sites of DNA methylation are occupied 
Table 1 | M. incognita putative orthologs for methyltransferases (DNMTs) and methyl-CpG binding domain (MBDs) proteins.

\begin{tabular}{|c|c|c|c|}
\hline Protein & Species & $\begin{array}{l}\text { Accession } \\
\text { number }\end{array}$ & $\begin{array}{l}\text { M. incognita putative } \\
\text { orthologs }\end{array}$ \\
\hline \multicolumn{4}{|c|}{ DNA METHYLTRANSFERASES (DNMTs) } \\
\hline DNMT-1 & $\begin{array}{l}\text { Trichinella } \\
\text { spiralis }\end{array}$ & EFV58204.1 & Minc01117 \\
\hline \multirow[t]{2}{*}{ DNMT-2 } & $\begin{array}{l}\text { Trichinella } \\
\text { spiralis }\end{array}$ & EFV60295.1 & $\begin{array}{l}\text { M. incognita absent } \\
\text { from OrthoMCL group }\end{array}$ \\
\hline & $\begin{array}{l}\text { Pristionchus } \\
\text { pacificus }\end{array}$ & AY766101.1 & \\
\hline DNMT-3 & $\begin{array}{l}\text { Trichinella } \\
\text { spiralis }\end{array}$ & EFV54759.1 & $\begin{array}{l}\text { M. incognita absent } \\
\text { from OrthoMCL group }\end{array}$ \\
\hline \multicolumn{4}{|c|}{ METHYL-CpG BINDING DOMAIN 2 (MBDs) } \\
\hline Cel-MBD-2 & $\begin{array}{l}\text { Caenorhabditis } \\
\text { elegans }\end{array}$ & C27A12.10 & Minc14299 Minc14778 \\
\hline Cbr-MBD-2 & $\begin{array}{l}\text { Caenorhabditis } \\
\text { briggsae }\end{array}$ & CBP11474 & \\
\hline Ppa-MBD-2 & $\begin{array}{l}\text { Pristionchus } \\
\text { pacificus }\end{array}$ & AY766102.1 & \\
\hline
\end{tabular}

by various proteins, including MBD proteins, which recruit the enzymatic machinery to establish silent chromatin. Nematodes such as C. elegans (Cel-MBD-2, accession number C27A12.10), C. briggsae (Cbr-MBD-2, accession number CBP11474) and $P$. pacificus (Ppa-MBD-2, accession numberAY766102.1) all contain an mbd-2-like gene (Gutierrez and Sommer, 2004). We found that two predicted M. incognita proteins, Minc14299 and Minc14778, could be orthologs to the MBD-2 like protein.

\section{STEP 2: CpG OBSERVED/EXPECTED RATIO (CpGO/e) ANALYSIS}

To evaluate the degree of DNA methylation, we calculated the empirical distribution of $\mathrm{CpGo/e}$ ratios for $M$. incognita transcriptome. A total of 63,838 expressed sequenced tags (EST) of M. incognita that were downloaded from NCBI-dbEST public database (Supplementary Material), filtered by size ( $\geq 500 \mathrm{bp}$ ) leading to 41,649 sequences of which 5000 were randomly chosen for $\mathrm{CpG}$ observed/expected ratio (CpGo/e) analysis. Most of the data came from $M$. incognita strain Morelos maintained at INRA Sophia Antipolis and recently published (Abad et al., 2008; Jaouannet et al., 2012). To validate the robustness of $\mathrm{CpGo/e}$ ratio method, the same experiment was conducted on $C$. elegans (initially 8722 ESTs), the model nematode lacking DNA methylation process, D. melanogaster (38,110 ESTs) and on A. mellifera (10,157 ESTs). D. melanogaster has been previously described for its lack of DNA methylation at late stages of development (Urieli-Shoval et al., 1982; Lyko et al., 2000) while DNA methylation occurs in A. mellifera (Elango et al., 2009). The CpGo/e ratio was used as a proxy to estimate the intragene DNA methylation content as previously described in Biomphalaria glabrata (Fneich et al., 2013). In C. elegans, the CpGo/e ratio profile exhibited an unskewed Gaussian distribution with an estimated mean value close to $1(0.93 \pm 0.26)$ as expected for species lacking such process (Figure 1A). A similar result was obtained with
D. melanogaster late stage ESTs (Estimated mean value $0.92 \pm$ 0.17; Figure 1B). In contrast, the $\mathrm{CpGo/e}$ ratio in A. mellifera transcripts can be approximated by a mixture of two Gaussian distributions (Figure 1C). This pattern has also been described in the mollusc B. glabrata where transcripts are divided in low and high methylated genes (Fneich et al., 2013). M. incognita CpGo/e ratio profile did not perfectly fit $C$. elegans profile (Figure 1D). In $M$. incognita, the $\mathrm{CpGo/e}$ ratio profile also exhibited a skewed near-Gaussian distribution but with a slight shift on the left compared to C. elegans profile. The estimated mean value for M. incognita transcriptome was inferior to $1(0.77 \pm 0.25)$. However, careful analysis of the curve fitting suggests the presence of two populations of sequences: one with a mean value around 0.9 and another with a mean value around 0.7 . These results could indicate the presence of two populations of genes in M. incognita: one with no methylation and one with very low methylation.

\section{STEP 3: GLOBAL DNA METHYLATION ANALYSIS BY LC-MS/MS}

Genomic DNA used for liquid chromatography-mass spectrometry analysis was prepared from $M$. incognita eggs with the CTAB method (Winnepenninckx et al., 1993) and stored at $-80^{\circ} \mathrm{C}$ until use. The levels of 5 -methylcytosine $(5-\mathrm{mC})$ and 5 -hydroxy methylcytosine $(5-\mathrm{hmC})$ were determined by liquid chromatography-mass spectrometry LC-MS/MS analysis as their deoxyribonucleosides as described in (Fneich et al., 2013). Neither 5-methyl-cytosine nor 5-hydrox-methyl-cytosine could be detected.

Taken together, our results show that DNA methylation is absent or very low in $M$. incognita.

\section{ANALYSIS OF NUCLEOSOMES, HISTONES, AND HISTONE MODIFICATIONS}

In the absence of DNA methylation, PTMs could represent one of the primary epigenetic transcriptional control mechanisms. Such a process has been shown to play a key role in the regulation of gene transcription of parasites, e.g., the apicomplexan family of protozoa and the metazoan Schistosoma (Mourão et al., 2012; Perrin et al., 2013). The importance of these modifications in regulating diverse developmental programs in parasites motivated us to study their importance in $M$. incognita in the framework of this study. Experiments were performed on M. incognita strain "Morelos" which have been previously used for the whole genome sequencing of this nematode (Abad et al., 2008). Because $M$. incognita is an obligatory plant parasite, it has to be maintained on plants to complete its life cycle. We used tomatoes, Solanum esculentum cultivar Saint Pierre, grown at $20^{\circ} \mathrm{C}$ in a greenhouse as $M$. incognita host for this study. Moreover, samples that we collected are considered to be clonal because of the mitotic parthenogenetic mode of reproduction of $M$. incognita and since the lab population was originated from the progeny of a single female. To grow and produce nematodes, one-monthold tomato plants are inoculated with $M$. incognita second-stage juveniles (J2s). As previously described by Rosso et al., 1999, eggs were collected from 7 week-old infected tomato roots after grinding, sterilizing $(0.5 \% \mathrm{NaOCl})$ and filtering steps. Extracted eggs were kept either to hatch $\mathrm{J} 2 \mathrm{~s}$ or to be aliquoted and stored at $-80^{\circ} \mathrm{C}$ for further experiments (e.g., genomic DNA extraction 

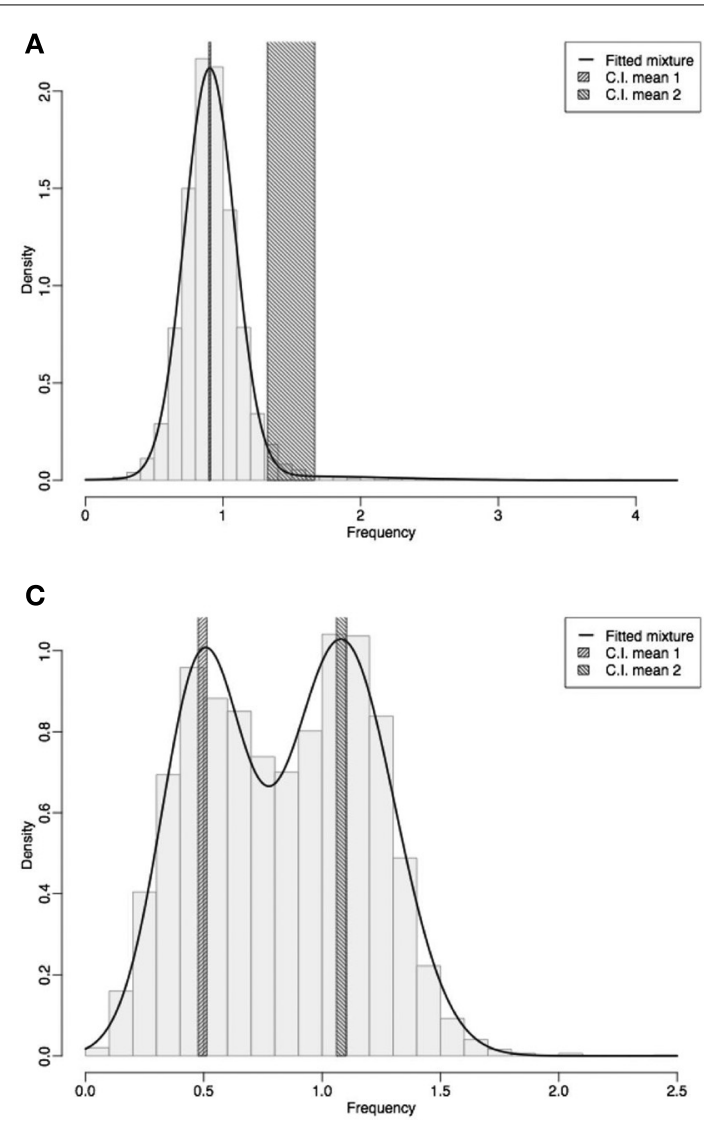

FIGURE 1 | Frequency distribution of CpG observed/expected ratio (CpGo/e) in four different species. (A) C. elegans and (B) D. melanogaster, and on (C) $A$. melifera and (D) $M$. incognita. CpGo/e ratio was measured as a proxy to estimate the $\mathrm{CpG}$ methylation in transcripts from EST data. $\mathrm{X}$ axis:

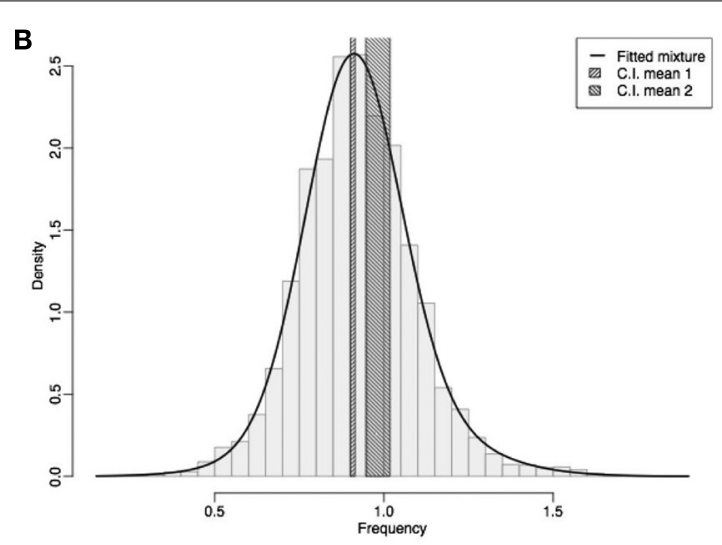

D

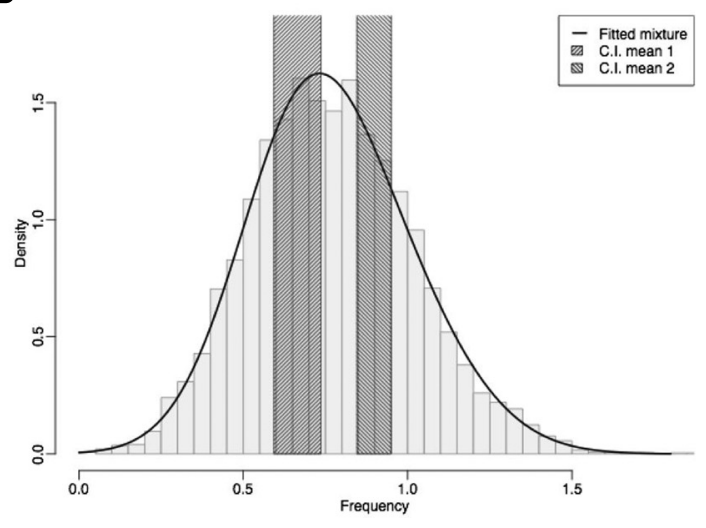

$\mathrm{CpGo/e}$ ratio, Y-axis density (frequency distribution) of ESTs. The figure displays a histogram of Bg GUA CpGo/e ratios with a fitted mixture distribution. The gray shaded bars represent $95 \%$ confidence intervals for the two mean values. and chromatin immuno-precipitation). To produce J2s, extracted eggs were kept on $10 \mu \mathrm{m}$ sieve in autoclaved tap water, aerated with an air pump, at room temperature. After 5 days, J2s hatched and were collected by centrifugation ( $13000 \mathrm{~g}, 1 \mathrm{~min})$. J2 samples were stored at $-80^{\circ} \mathrm{C}$ for further experiments (e.g., Western blot and chromatin immuno-precipitation).

\section{STEP 1: DETECTION OF CANONICAL HISTONES AND HISTONE MODIFICATIONS BY WESTERN BLOTS}

The nucleosome is subject to a dizzying array of posttranslational modifications, which work alone or in combination to constitute a histone code that regulates chromatin structure and function (Jenuwein and Allis, 2001). The four core histones, H2A, H2B, $\mathrm{H} 3$, and $\mathrm{H} 4$, which are responsible for folding DNA into nucleosomes, have been described as very highly conserved throughout evolution (Mariño-Ramírez et al., 2006). This high conservation allowed us to test commercial antibodies that react with mammals but also with a wide range of species. In some species, chromatin is condensed around the centromere and characterized by several methyl groups on lysine amino acid (K) at 9 and 20 position of histone $\mathrm{H} 3$ and $\mathrm{H} 4$ respectively. These marks are noted H3K9Me2/3 and H4K20Me3 and can be recognized by specific antibodies. Before immuno-precipitation of $M$. incognita DNA, we tested 12 commercial antibodies, directed against histone H3 and several $\mathrm{H} 3$ and $\mathrm{H} 4$ modifications, on M. incognita J2s with western blots.

Western blots were performed as previously described by Azzi et al. (2009). Briefly, about one thousand M. incognita J2s were re-suspended in denaturation buffer, treated by sonication and boiled $5 \mathrm{~min}$ at $99^{\circ} \mathrm{C}$. Proteins were separated by SDS-PAGE and transferred to nitrocellulose membranes (Amersham RPN203D) by the semi-dry method (SEMI-PHOR Bio-Rad). The membrane was blocked overnight at $4^{\circ} \mathrm{C}$ in blocking buffer and incubated with one of the 12 following commercial antibodies $(1 \mu \mathrm{g} / \mu \mathrm{l})$ : Anti-histone $\mathrm{H} 3$ (H3, abcam ab1791 and Active Motif 39164); Anti-Histone H3 dimethylated at lysine $4(\mathrm{H} 3 \mathrm{~K} 4 \mathrm{Me} 2$, Abcam ab32356) or at lysine 36 (H3K36Me2, Abcam ab9049); Anti-Histone H3 trimethylated at lysine 4 (H3K4Me3, Millipore 04-745 and Abcam ab8580), at lysine 9 (H3K9Me3, Abcam ab8898 and Upstate) or at lysine 27 (H3K27Me3, Diagenode pAB-069-050); Anti-Histone $\mathrm{H} 3$ acetylated at lysine 9 (H3K9Ac, Upstate); Anti-Histone H4 trimethylated at lysine 20 (H4K20Me3, abcam ab9053) and Antihyperacetylated Histone $\mathrm{H} 4$ (H4PentaAc, Upstate). Bands were 
revealed by Enhanced Chemical Luminescence (ECL Pierce) and direct exposure to X-ray film (Amersham).

The antibodies should give a unique staining on the membrane (one unique band) to be selected for DNA immunoprecipitation. Three antibodies, Anti-H3K4Me3 (Abcam), Anti-H3K9Me3 (Abcam and Upstate), did not give any signal (Supplementary Figure 2A). One antibody, Anti-H4PentaAc gave a non-specific signal with many high molecular weight bands (Supplementary Figure 2B). Two antibodies, AntiH3K27Me3 and Anti-H3K36Me2 gave two distinct bands (Supplementary Figure 2C). Six antibodies, Anti-histone H3 (Abcam and Active), Anti-H3K4Me2, Anti-H3K4Me3 (Millipore), Anti-H3K9Ac and Anti-H4K20Me3 gave a unique signal at $17 \mathrm{kDa}$ (Figure 2) as expected for histones (Bártová et al., 2005).

We conclude that canonical histones and histone modifications are present and that six commercially available antibodies could be used for immuno-precipitation of $M$. incognita chromatin.

\section{STEP 2: CONTROLLED FRAGMENTATION INTO NUCLEOSOMES}

Because little is known about chromatin organization in M. incognita, we started by testing partial Micrococcal Nuclease (MNase) digestion of chromatin to reveal its nucleosome structure, which is the fundamental unit of chromatin. Because DNA portions of nucleosome core particles are less accessible for MNase than linking sections, DNA gets digested into fragments of lengths equal to multiplicity of distance between mono-nucleosomes. First, to access nuclei, we tested different grinding times, from 3 to $10 \mathrm{~min}$, for cell lysis in Dounce homogenizer. As a measure for grinding efficiency, we colored nuclei using Hoechst, a fluorescent stain that binds strongly DNA. Also, because M. incognita life-stages are really different in shape and composition, we tested these grinding conditions on both J2s and eggs. Best results were obtained with $10 \mathrm{~min}$ total in Dounce homogenizer, distributed as $5 \mathrm{~min}$ grinding following by $5 \mathrm{~min}$ at rest (Figure 3A). Then, we tested different MNase concentrations (from 15 to $30 \mathrm{U}$; Figure 3B and Supplementary Figure 3) and incubation times $(0,2,4,5,6$, and $7 \mathrm{~min})$. Typical mono-nucleosome structure was observed with fragments about $150 \mathrm{bp}$ and multiplicity of this size (Figure 3B). Finally, $5 \mathrm{~min}$ incubation with $1 \mu \mathrm{l}$ MNase (15 U) was chosen as the standard conditions to perform further steps of immuno-precipitation of the chromatin in M. incognita.

\section{STEP 3: CHROMATIN IMMUNO-PRECIPITATION (ChIP)}

To immunoprecipitate $M$. incognita DNA, we used the ChIP procedure adapted for non-model organisms by Cosseau et al. (2009). Each step of the procedure (Supplementary Material) had to be customized for M. incognita. Basically, we tested different grinding times for cell lysis and different Micrococcal nuclease (MNase, Affymetrix, USB 70196Y) concentrations and incubation times (see Results). Nuclei were stained with Hoechst 33342 fluorescent stain (1/2000 dilution; Invitrogen H1399) and observed under microscope (40X, Nikon T1-SM) before and after grinding.

Because the antibody should be in excess over the histone of interest for ChIP, we tested protein/chromatin precipitation efficiency in $M$. incognita with increasing antibody volume ranging from 0 to $16 \mu \mathrm{l}(1 \mu \mathrm{g} / \mu \mathrm{l})$, for each of 3 tested antibodies (among the 6 antibodies that could be use): Anti-H3 (Abcam), AntiH3K4Me3 (Millipore), and Anti-H4K20Me3. For each antibody concentration, DNA from the bound and unbound fractions

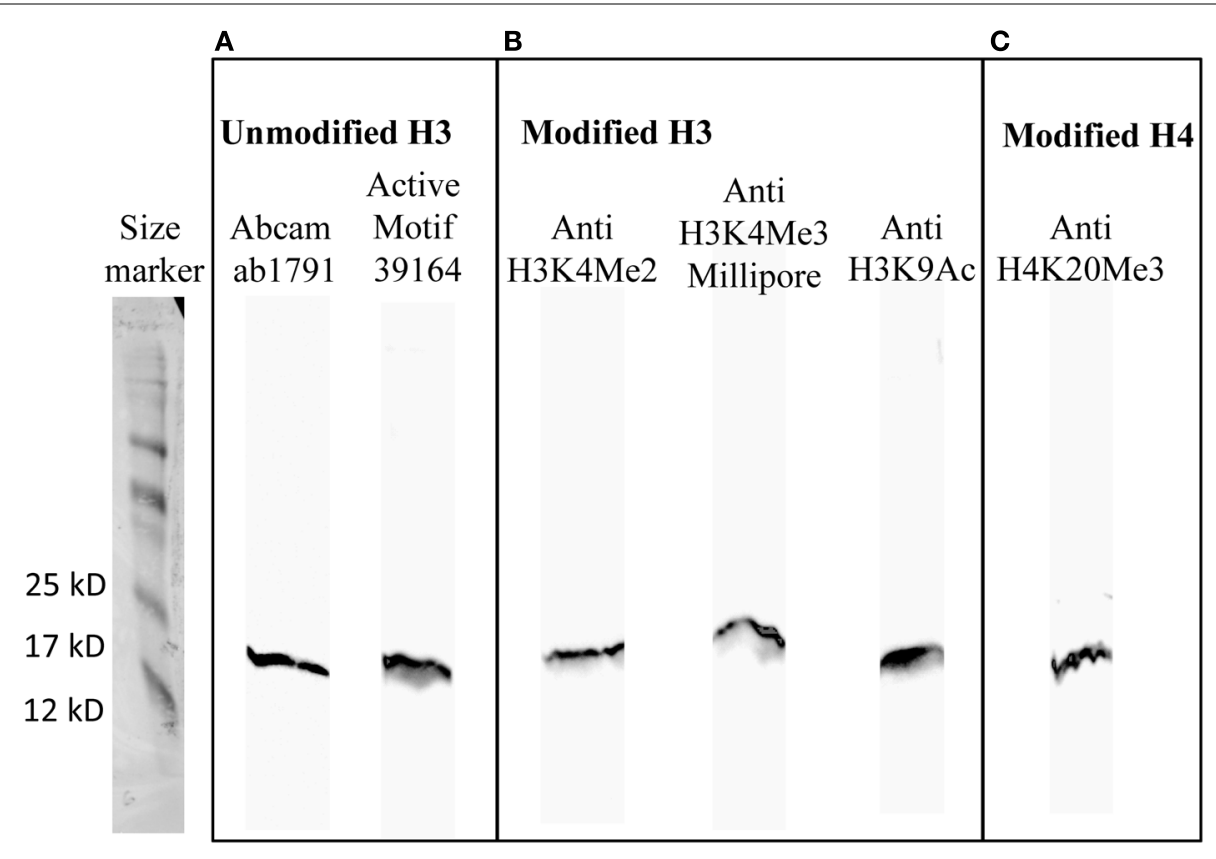

FIGURE 2 | Western blot detection of (A) unmodified histone H3, (B) modified histone H3: dimethylated H3 at lysine 4 (H3K4Me2) and acetylated H3 at lysine 9 (H3K9Ac) and (C) modified histone H4: trimethylated $\mathrm{H} 4$ at lysine 20 (H4K2OMe3) in M. incognita J2s. 
was extracted with phenol/chloroform so we could quantify the sequences associated with each antibody by qPCR. In other species, histone marks such as $\mathrm{H} 3 \mathrm{~K} 4 \mathrm{Me} 3$ were enriched around the transcription start sites (TSSs) of genes and were associated with active transcription (Du et al., 2013). For this reason, primers were designed to match genomic region close to TSSs of

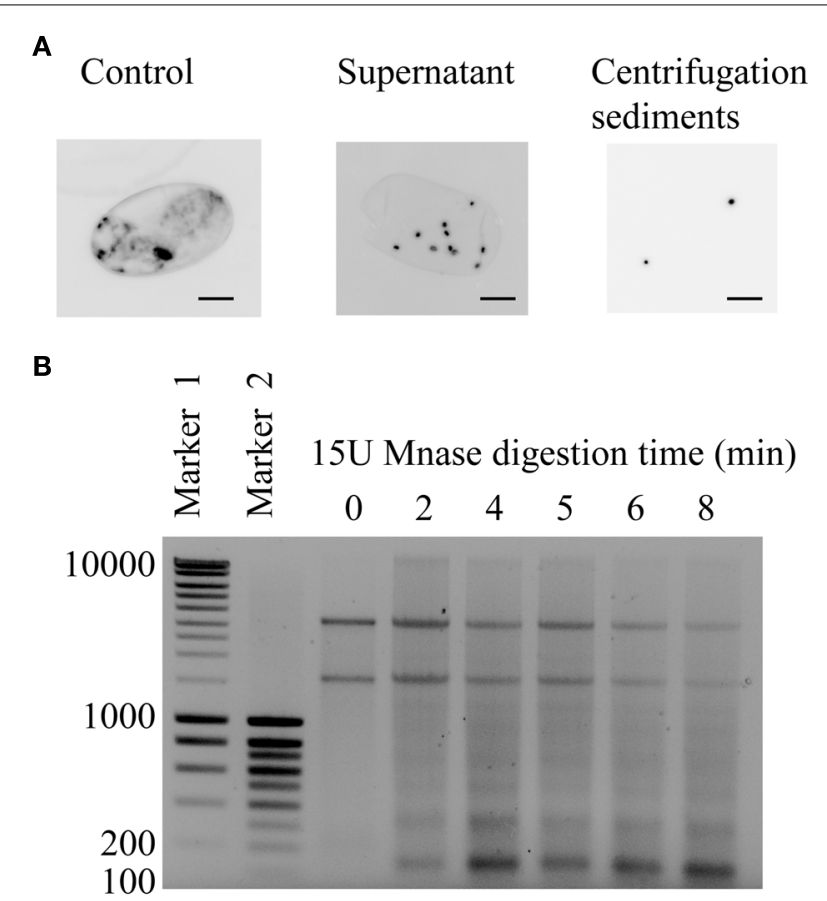

FIGURE 3 | (A) Pictures showing nucleus (in black; Hoechst staining) of $M$. incognita eggs observed under microscope (40X, Nikon T1-SM) before (Control) and after grinding (Supernatant and Centrifugation sediments). The experiment started with intact eggs (Control) that were grinded for $5 \mathrm{~min}$ in Dounce homogenizer to release nuclei. After centrifugation, only nuclei were observed in centrifugation sediments whereas nuclei and fragments remained in supernatant. Scale bar, $10 \mu \mathrm{m}$. (B) Electrophoresis gel of $M$. incognita eggs DNA after $0,2,4,5,6$, and 7 min MNase (15 U) treatment showing a very characteristic pattern, similar to a ladder, for nucleosome. Marker 1 and 2 exhibit regularly spaced bands ranging from 200 to $10,000 \mathrm{bp}$ and 100 to $1000 \mathrm{bp}$, respectively.
M. incognita housekeeping genes, Actin and GAPDH (Minc06773 and Minc12412, respectively; Table 2). Quantitative Real-time PCR (qPCR) analyses were performed using the LightCycler 2.0 system (Roche Applied Science) and LightCycler Faststart DNA Master SYBR Green I kit (Roche Applied Science). qPCR amplification was done in a final volume of $10 \mu \mathrm{l}$ composed of $2.5 \mu \mathrm{l}$ of immuno-precipitated chromatin, $1.5 \mu \mathrm{l} \mathrm{H}_{2} \mathrm{O}, 0.5 \mu \mathrm{l}$ of each primer $10 \mu \mathrm{M}$ and $5 \mu \mathrm{l}$ of master mix. The following Light-Cycler run protocol was used: denaturation, $95^{\circ} \mathrm{C}, 10 \mathrm{~min}$; amplification and quantification (repeated 40 times), $95^{\circ} \mathrm{C}$ for $10 \mathrm{~s}, 62^{\circ} \mathrm{C}$ for $5 \mathrm{~s}, 72^{\circ} \mathrm{C}$ for $16 \mathrm{~s}$; melting curve, $60-95^{\circ} \mathrm{C}$ with a heating rate of $0.11^{\circ} \mathrm{C} / \mathrm{s}$ and continuous fluorescence measurement, and a cooling step to $40^{\circ} \mathrm{C}$. For each reaction, the cycle threshold (Ct) was determined using the " 2 nd derivative" method of the LightCycler 480 Software release 1.5. Primers were designed for this experiment with the Primer3 web-interface (Table 2). Quality and specificity controls were performed for each qPCR product (Supplementary Figure 1): amplification of a unique $150 \mathrm{bp}$ band was verified by electrophoresis separation through a $2 \%$ agarose gel; amplicons were sequenced to verify that they matched expected sequence (GATC-biotech; Table 2); Primer efficiency was calculated based on $C t$ value for increasing genomic DNA concentration (from 0 to $1 \mathrm{ng} / \mathrm{ul}$ ); Melting curve and negative first derivative of the melting-curve were recorded for each qPCR product to validate that only one product was amplified (one peak was observed).

The amount of target DNA recovered in the immuneprecipitated fraction was quantified by calculating the percent input recovery (\% IR) normalized with the percent input recovery obtained with the housekeeping gene as previously described by Cosseau et al. (2009).

Also, because of previous MNase digestion, the amplicon size has to be no longer than $150 \mathrm{pb}$ to match $M$. incognita mono-nucleosome length. With $M$. incognita DNA, antibodies Anti-H3 and Anti-H4K20Me3 never reached saturation up to $16 \mu l$ (Figure 4). This could mean that these antibodies are not specific to histone $\mathrm{H} 3$ and histone $\mathrm{H} 4$ trimethylated at lysine 20 but recognize other proteins/marks. So, the more antibody was added, the more proteins interacted which generated a background signal detected thanks to qPCR. On the contrary, the Anti-H3K4Me3 antibody successfully immuno-precipitated $M$.

Table 2 | Amplicon sequences and primer sequences designed for qPCR.

\begin{tabular}{|c|c|c|c|}
\hline Name & Sequence & Length (bp) & Product size (bp) \\
\hline M. incognita & \multirow{2}{*}{\multicolumn{3}{|c|}{$\begin{array}{l}\text { CGTGCAGCGGTTGAGAAGGATACTGTCCAAGTTGTTGCTGTCAATGACCCGTTCATTGATCTTGACTATATGGTTTGGGGGAGACTTT } \\
\text { CTATTAATAACTCCAATAACTTTTAGGTCTATATGTTTAACTATGATTCCACCCACGGACGC }\end{array}$}} \\
\hline GAPDH amplicon & & & \\
\hline Mi_ChIP_GAPDH_F & CGTGCAGCGGTTGAGAAGGA & 20 & \multirow[t]{2}{*}{150} \\
\hline Mi_ChIP_GAPDH_R & GCGTCCGTGGGTGGAATCAT & 20 & \\
\hline Mi_ChIP_Actin_F & AAGATGGATGAAGAGGTAGCCGCCC & 25 & \multirow[t]{2}{*}{150} \\
\hline Mi_ChIP_Actin_R & TGGAAAAACGGCACGAGGAGCA & 22 & \\
\hline
\end{tabular}



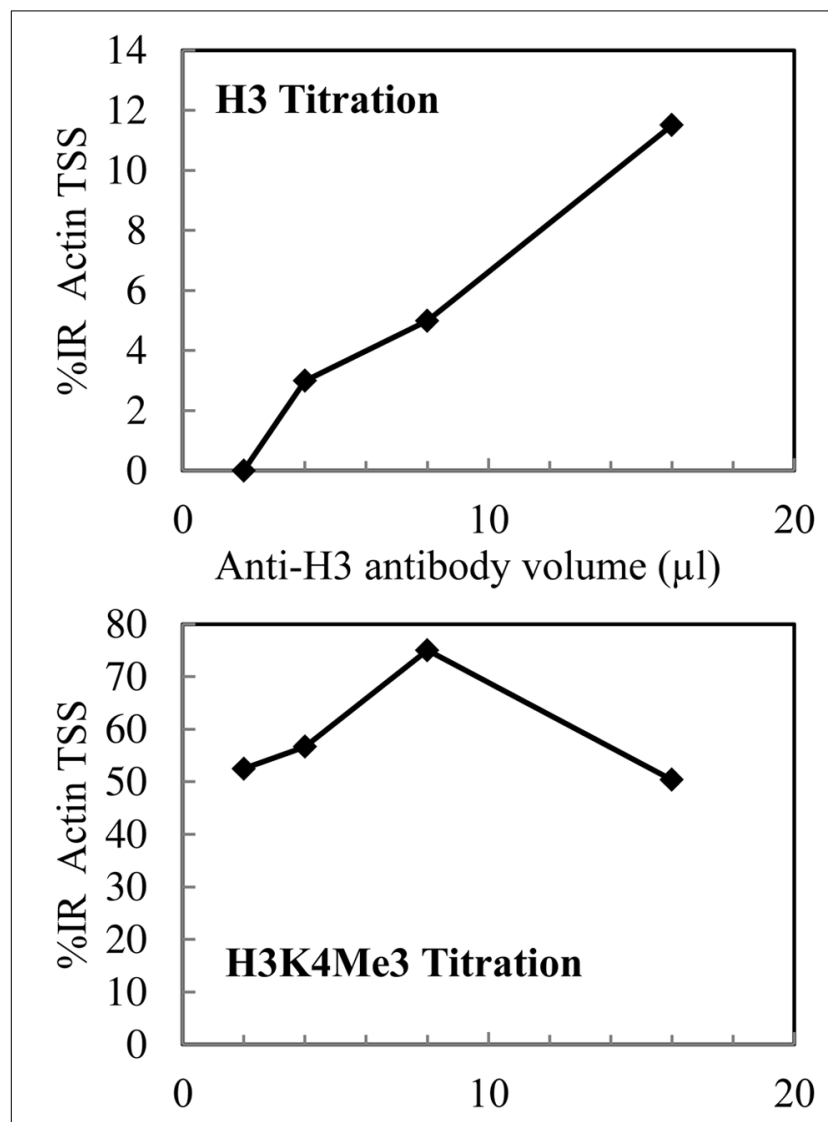

Anti-H3K4Me3 antibody volume $(\mu 1)$

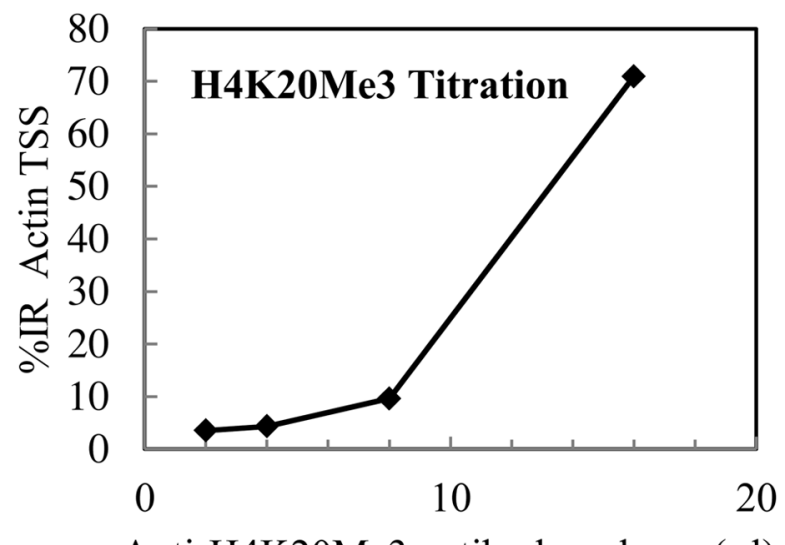

Anti-H4K20Me3 antibody volume $(\mu \mathrm{l})$

FIGURE 4 | qPCR on $M$. incognita chromatin that had been immuno-precipitated with various volumes (0-16 $\mu \mathrm{l})$ of Anti-H3,

Anti-H3K4Me3, and Anti-H4K20Me3 antibodies $(1 \mu \mathrm{g} / \mu \mathrm{I})$. The amount of target DNA recovered in the immune-precipitated fraction was quantified by calculating the percent input recovery (\% IR) normalized with the percent input recovery obtained with the housekeeping gene as previously described by Cosseau et al. (2009).

incognita chromatin and reached saturation at $8 \mu$ l (Figure 4). Titration saturation for higher antibody concentrations indicates that the antibody is specific to the histone mark (Cosseau et al., 2009). Therefore, this antibody could specifically immuno-precipitate $M$. incognita and could be use for further analysis.

To summarize, we optimized the ChIP protocol for M. incognita, based on the previously published method for non-model organisms (Cosseau et al., 2009). First, we have optimized lysis and extraction of nuclei for $M$. incognita to $10 \mathrm{~min}$ grinding in digestion buffer. Then we found that the optimum time for MNase digestion (15U), to obtain fragmented chromatin into mono-nucleosome to penta-nucleosomes, a size range that is optimal for ChIP, was $5 \mathrm{~min}$. Finally, qPCR experiments indicated that N-Chip procedure should be performed with an excess of $8 \mu \mathrm{l}$ antibody Anti- H3K4Me3. Now that chromatin immunoprecipitation has been optimized in M. incognita it opens new perspectives to further investigate the genome-wide distribution of PTMs by high-throughput sequencing (N-ChIP-Seq).

\section{DISCUSSION}

In the past few years, genome projects have demonstrated that DNA methylation is far more widespread than one would expect from the lack of DNA methylation in non-mammalian model organisms, such as the model nematode C. elegans. Moreover, functional DNA methylation has been recently reported in the parasitic nematode T. spiralis (Gao et al., 2012, 2014). Altogether, this makes us wonder what DNA methylation picture in $M$. incognita could be.

The de novo DNA methylation process can produce new methylated sites without pre-existing pattern by affecting cytosine nucleotide (Goll and Bestor, 2005). Depending on CG density, some of these de novo sites can then be maintained by DNMT maintenance methyltransferases. $\mathrm{CpG}$ under representation is generally seen as sign of DNA methylation (Bird, 1980). CpG o/e suggests that DNA methylation was present as a subset of genes in M. incognita ancestral species, that these sequences were obtained by horizontal gene transfer from a species with DNA methylation or that the EST database was contaminated. In the first case, $M$. incognita might have lost DNA methylation enzymes, which may have been present in the ancestral nematode, from its genome, as what is thought for C. elegans (Gutierrez and Sommer, 2004; Suzuki and Bird, 2008). Nevertheless, the observed CpG bias could also be due to other mechanisms (Jabbari and Bernardi, 2004). By LC-MS DNA methylation could not be detected in $M$. incognita that goes in line with the absence of gene coding for de novo DNA methylation activity.

The nucleosome is one of the most highly conserved structures known in eukaryotes, which is consistent with the particle's fundamental role in packaging DNA into the nucleus (Turner, 1993, 2005). After prolonged digestion, the MNase degrades the chromatin to its fundamental repeating unit, called the nucleosome. In M. incognita, DNA fragment observed miccrococal nuclease digestion of $150 \mathrm{bp}$ define the nucleosome that is consistent with what is known of the "primary structure" of chromatin with DNA fragment of length around the $\sim 145-150 \mathrm{pb}$ (Kornberg and Thomas, 1974; Luger et al., 1997; Rando, 2011). However, it is becoming clear that chromatin structures are not nearly as uniform and regular as previously assumed: nucleosomes vary in their histone protein components due to the incorporation of variant histones and PTMs (Luger et al., 2012; 
Turner, 2012). All PTMs, reversible for the most part, either change nucleosome structure directly by affecting histone proteins and DNA interactions or indirectly by recruiting binding proteins that act on the underlying chromatin structure, as has been proposed in the "histone code" hypothesis (Strahl and Allis, 2000). However, in spite of the high conservation of histone proteins along evolution, their degree of modification varies enormously according to the species, the developmental stage, the tissue and the cellular cycle phase (Strahl and Allis, 2000; Trojer and Reinberg, 2007; Luger et al., 2012; Turner, 2012). Here, we provided evidences for a "canonical nucleosome structure" in $M$. incognita with recognition of both unmodified and modified histone $\mathrm{H} 3$ as well as modified histone $\mathrm{H} 4$. Both euchromatin and heterochromatin regions contain specific PTMs and binding proteins. For instance, in C. elegans, heterochromatin is enriched in trimethylated $\mathrm{H} 3$ histones at lysine 56 (H3K56Me3) and lysine 9 (H3K9Me3; Jack et al., 2013). Ultimately, to identify features of transcriptional networks that regulate developmental processes, ChIP-sequencing was completed genome-wide in C. elegans (Niu et al., 2011). We expect to build on this foundation, together with this preliminary study, for further evaluation of transcriptional regulatory mechanisms during $M$. incognita development.

In addition, it is to be noted that nematodes could be divided in monocentric species and holocentric species (Subirana and Messeguer, 2013). C. elegans as well as M. incognita belong to the latter in which the centromeric function spreads out over the whole chromosomes (Triantaphyllou, 1983; Dernburg, 2001; Melters et al., 2012). Moreover, changes in chromosome number and structural rearrangements have been reported in Meloidogyne species (Triantaphyllou, 1981; Castagnone-Sereno, 2006; Abad et al., 2008). Being able to study position and histone protein components of $M$. incognita nucleosomes can be used to better understand diverse biological processes including DNA replication, recombination, mutation and repair. Because of the intimate relationship between nucleosome locations and these processes, we anticipate that ChIP-seq experiments in $M$. incognita will shed more light on genome evolution that seems to be crucial for an organism whose mode of reproduction is obligatory mitotic parthenogenesis (apomixis).

One major role for epigenetic variation in evolution would be to promote phenotypic variability, and allow populations to widely explore new environmental conditions. However, the detailed mechanisms thanks to which parasites evade host immunity for instance are poorly understood. M. incognita constitutes an ideal model to study these mechanisms, especially as inheritance of the nematode virulence does not segregate in accordance with Mendel's laws together with asexual multiplication in host plants (Castagnone-Sereno et al., 1994). Because clones can be obtained, genetic variability is reduced. As a result, $M$. incognita provides support for the study of epigenetic heredity distinct from the canonical Mendelian rules. Recently, it became apparent that parasitism success is partly accomplished by epigenetic means in several parasites, including, among others, protozoans Plasmodium and trematodes Schistosoma. More especially, PTMs plays a key role in the regulation of gene transcription of these parasites (Mourão et al., 2012; Perrin et al., 2013). For instance, it has been conclusively shown that PTMs, especially acetylations and methylations, and the propagation of heterochromatin away from the telomeres control the transcriptional switching between VAR genes that will trigger antigenic variation and enable immune evasion by the parasite, Plasmodium falciparum (Freitas-Junior et al., 2005; Chookajorn et al., 2007; Malmquist et al., 2012; Jiang et al., 2013). In Schistosoma mansoni, N-ChIP-seq experiments have been successfully performed to compare chromatin structure at different stages of the parasite life cycle, on different strains and on different sexes (Caby and Pierce, 2009; Cosseau et al., 2009; Lepesant et al., 2011, 2012). These advances in understanding S. mansoni epigenetic regulation have lead to the identification of promising new targets for the development of new drug to combat schistosomiasis (Pierce et al., 2012; Lancelot et al., 2013; Marek et al., 2013).

The importance of PTMs in regulating crucial developmental processes in parasites combined with the recent resolution of the genome sequences of $M$. incognita make the study of epigenetic machinery possible and susceptible to break new ground.

\section{CONCLUSION}

For the first time in M. incognita, this study details DNA methylation and nucleosome structure, carriers of epigenetic information. Genomic DNA of M. incognita is not methylated. The general characteristics of nucleosome structure that we observed in the $M$. incognita genome are in accordance to what epigenetic studies have reported from other invertebrate species. Histone modifications, important markers of function and chromatin state, were identified in $M$. incognita. This study opens the way for analyzing epigenetic mechanisms in $M$. incognita at a whole-genome scale to identify new biological processes involved in the generation of phenotypic variation in absence of sexual reproduction in an ecological and economical important model.

\section{AUTHOR CONTRIBUTIONS}

Conceived and designed the experiments: Céline Cosseau, Christoph Grunau, Laetitia Perfus-Barbeoch, Pierre Abad, Philippe Castagnone-Sereno. Performed the experiments: David Roquis, Laetitia Perfus-Barbeoch, Loris Pratx, Michael Reichelt, Sara Fneich. Analyzed the data: Céline Cosseau, Christoph Grunau, Laetitia Perfus-Barbeoch, Pierre Abad, Philippe Castagnone-Sereno. Wrote the paper: Céline Cosseau, Christoph Grunau, David Roquis, Laetitia Perfus-Barbeoch, Michael Reichelt, Pierre Abad, Philippe Castagnone-Sereno, Sara Fneich.

\section{ACKNOWLEDGMENTS}

The authors want to thank Nathalie Marteu (INRA, Sophia Antipolis, France) for growing plant and nematode materials, Etienne G. J. Danchin (INRA, Sophia Antipolis, France) for help with OrthoMCL analysis and Bruno Favery (INRA, Sophia Antipolis, France) for critical reading of the manuscript. This work was supported by the French Government (National Research Agency, ANR) through the "Investments for the Future" LABEX SIGNALIFE: program reference \# ANR-11-LABX-0028-0. 


\section{SUPPLEMENTARY MATERIAL}

The Supplementary Material for this article can be found online at: http://www.frontiersin.org/journal/10.3389/fphys. 2014.00211/abstract

Supplementary material : Links to public databases.

Expressed sequenced tags (EST) of $M$. incognita from NCBIdbEST: http://www.ncbi.nlm.nih.gov/nucest/?term=meloido gyne+incognita

Primer3 web-interface: www.bioinformatics.nl/primer3plus/

M. incognita whole proteome: http://www6.inra.fr/ meloidogyne_incognita/Genomic-resources/Blast

\section{Supplementary Figure 1 | Quality and specificity controls for qPCR analysis. (A) Electrophoresis gel of 150 bp genomic DNA amplified with primers specifically designed for ChIP validation. Markers 1 and 2 exhibit regularly spaced bands ranging from 200 to $10,000 \mathrm{bp}$ and 100 to $1000 \mathrm{bp}$, respectively. (B) Primer efficiency was assessed on increasing genomic DNA concentration (from 0 to $1 \mathrm{ng} / \mathrm{ul}$ ): increase in fluorescence intensity is proportional to the increase in amplicon concentration. (C) Melting curves and negative first derivative of the melting-curve: presence of a single homogeneous melt peak confirms specific amplification. \\ Supplementary Figure $\mathbf{2}$ | Western blots showing non-specific signals in M. incognita J2s. (A) Anti-H3K4Me3 or Anti-H3K9Me3 antibodies. (B) Anti-H4PentaAc antibody. (C) Anti-H3K27Me3 or Anti-H3K36Me2 antibodies.}

\section{Supplementary Figure 3 | Electrophoresis gel of $\boldsymbol{M}$. incognita eggs DNA after $\mathbf{0 , 2}, \mathbf{4}, \mathbf{5}, \mathbf{6}$, and $\mathbf{7} \mathbf{~ m i n ~} \mathbf{M N a s e}(\mathbf{3 0 U})$. Marker 1 and 2 exhibit regularly spaced bands ranging from 200 to $10,000 \mathrm{bp}$ and 100 to $1000 \mathrm{bp}$, respectively.}

\section{REFERENCES}

Abad, P., Gouzy, J., Aury, J. M., Castagnone-Sereno, P., Danchin, E. G., Deleury, E., et al. (2008). Genome sequence of the metazoan plant-parasitic nematode Meloidogyne incognita. Nat. Biotechnol. 26, 909-915. doi: 10.1038/nbt.1482

Azzi, A., Cosseau, C., and Grunau, C. (2009). Schistosoma mansoni: developmental arrest of miracidia treated with histone deacetylase inhibitors. Exp. Parasitol. 121, 288-291. doi: 10.1016/j.exppara.2008.11.010

Bártová, E., Pacherník, J., Harnicarová, A., Kovarík, A., Kovaríková, M., Hofmanová, J., et al. (2005). Nuclear levels and patterns of histone H3 modification and HP1 proteins after inhibition of histone deacetylases. J. Cell Sci. 118, 5035-5046. doi: 10.1242/jcs.02621

Bird, A. (1980). DNA methylation and the frequency of CpG in animal DNA. Nucleic Acids Res. 8, 1499-1504. doi: 10.1093/nar/8.7.1499

Caby, S., and Pierce, R. J. (2009). Quantitative chromatin immunoprecipitation (QChIP) applied to Schistosoma mansoni. Mol. Biochem. Parasitol. 166, 77-80. doi: 10.1016/j.molbiopara.2009.02.014

Caillaud, M. C., Dubreuil, G., Quentin, M., Perfus-Barbeoch, L., Lecomte, P., de Almeida Engler, J., et al. (2008). Root-knot nematodes manipulate plant cell functions during a compatible interaction. J. Plant Physiol. 165, 104-113. doi: 10.1016/j.jplph.2007.05.007

Castagnone-Sereno, P. (2006). Genetic variability and adaptive evolution in parthenogenetic root-knot nematodes. Heredity 96, 282-289. doi: 10.1038/sj.hdy.6800794

Castagnone-Sereno, P., Wajnberg, E., Bongiovanni, M., Leroy, F., and Dalmasso, A. (1994). Genetic variation in Meloidogyne incognita virulence against the tomato Mi resistance gene: evidence from isofemale line selection studies. Theor. Appl. Genet. 88, 749-753. doi: 10.1007/BF01253980

Chookajorn, T., Dzikowski, R., Frank, M., Li, F., Jiwani, A. Z., Hartl, D. L., et al. (2007). Epigenetic memory at malaria virulence genes. Proc. Natl. Acad. Sci. U.S.A. 104, 899-902. doi: 10.1073/pnas.0609084103

Cosseau, C., Azzi, A., Smith, K., Freitag, M., Mitta, G., and Grunau, C. (2009). Native chromatin immunoprecipitation (N-ChIP) and ChIP-Seq of Schistosoma mansoni: Critical experimental parameters. Mol. Biochem. Parasitol. 166, 70-76. doi: 10.1016/j.molbiopara.2009.02.015

Danchin, E. G., Rosso, M. N., Vieira, P., de Almeida-Engler, J., Coutinho, P. M., Henrissat, B., et al. (2010). Multiple lateral gene transfers and duplications have promoted plant parasitism ability in nematodes. Proc. Natl. Acad. Sci. U.S.A. 107, 17651-17656. doi: 10.1073/pnas. 1008486107

Dernburg, A. F. (2001). Here, there, and everywhere: kinetochore function on holocentric chromosomes. J. Cell Biol. 153, F33-F38. doi: 10.1083/jcb.153.6.F33

Du, Z., Li, H., Wei, Q., Zhao, X., Wang, C., Zhu, Q., et al. (2013). Genome-wide analysis of histone modifications: $\mathrm{H} 3 \mathrm{~K} 4 \mathrm{me} 2, \mathrm{H} 3 \mathrm{~K} 4 \mathrm{me}$, H3K9ac, and H3K27ac in Oryza sativa L. Japonica. Mol. Plant. 6, 1463-1472. doi: 10.1093/mp/sst018

Elango, N., Hunt, B. G., Goodisman, M. A., and Yi, S. V. (2009). DNA methylation is widespread and associated with differential gene expression in castes of the honeybee, Apis mellifera. Proc. Natl. Acad. Sci. U.S.A. 106, 11206-11211. doi: 10.1073/pnas.0900301106

Fneich, S., Dheilly, N., Adema, C., Rognon, A., Reichelt, M., Bulla, J., et al. (2013). 5-methyl-cytosine and 5-hydroxy-methyl-cytosine in the genome of Biomphalaria glabrata, a snail intermediate host of Schistosoma mansoni. Parasit. Vectors 6:167. doi: 10.1186/1756-3305-6-167

Freitas-Junior, L. H., Hernandez-Rivas, R., Ralph, S. A., Montiel-Condado, D., Ruvalcaba-Salazar, O. K., Rojas-Meza, A. P., et al. (2005). Telomeric heterochromatin propagation and histone acetylation control mutually exclusive expression of antigenic variation genes in malaria parasites. Cell. 121, 25-36. doi: 10.1016/j.cell.2005.01.037

Gao, F., Liu, X., Wu, X. P., Wang, X. L., Gong, D., Lu, H., et al. (2012). Differential DNA methylation in discrete developmental stages of the parasitic nematode Trichinella spiralis. Genome Biol. 13, R100. doi: 10.1186/gb-2012-13-10-r100

Gao, F., Wang, R., and Liu, M. (2014). Trichinella spiralis, potential model nematode for epigenetics and its implication in metazoan parasitism. Front. Physiol. 4:410. doi: 10.3389/fphys.2013.00410

Goll, M. G., and Bestor, T. H. (2005). Eukaryotic cytosine methyltransferases. Annu. Rev. Biochem. 74, 481-514. doi: 10.1146/annurev.biochem.74.010904.153721

Gómez-Díaz, E., Jordà, M., Peinado, M. A., and Rivero, A. (2012). Epigenetics of host-pathogen interactions: the road ahead and the road behind. PLoS Pathog. 8:e1003007. doi: 10.1371/journal.ppat.1003007

Gutierrez, A., and Sommer, R. J. (2004). Evolution of dnmt-2 and mbd-2like genes in the free-living nematodes Pristionchus pacificus, Caenorhabditis elegans and Caenorhabditis briggsae. Nucleic Acids Res. 32, 6388-6396. doi: 10.1093/nar/gkh982

Hendrich, B., and Tweedie, S. (2003). The methyl-CpG binding domain and the evolving role of DNA methylation in animals. Trends Genet. 19, 269-277. doi: 10.1016/S0168-9525(03)00080-5

Jabbari, K., and Bernardi, G. (2004). Cytosine methylation and CpG, TpG (CpA) and TpA frequencies. Gene 333, 143-149. doi: 10.1016/j.gene.2004.02.043

Jablonka, E., and Lamb, M. J. (2002). The changing concept of epigenetics. Ann. N. Y. Acad. Sci. 981, 82-96. doi: 10.1111/j.1749-6632.2002.tb04913.x

Jack, A. P., Bussemer, S., Hahn, M., Pünzeler, S., Snyder, M., Wells, M., et al. (2013). H3K56me3 is a novel, conserved heterochromatic mark that largely but not completely overlaps with $\mathrm{H} 3 \mathrm{~K} 9 \mathrm{me} 3$ in both regulation and localization. PLoS ONE 8:e51765. doi: 10.1371/journal.pone.0051765

Jaouannet, M., Perfus-Barbeoch, L., Deleury, E., Magliano, M., Engler, G., Vieira, P., et al. (2012). A root-knot nematode-secreted protein is injected into giant cells and targeted to the nuclei. New Phytol. 194, 924-931 doi: 10.1111/j.14698137.2012.04164.x

Jenuwein, T., and Allis, C. D. (2001). Translating the histone code. Science 293, 1074-1080. doi: 10.1126/science. 1063127

Jiang, L., Mu, J., Zhang, Q., Ni, T., Srinivasan, P., Rayavara, K., et al. (2013). PfSETvs methylation of histone H3K36 represses virulence genes in Plasmodium falciparum. Nature 499, 223-227. doi: 10.1038/nature12361

Kornberg, R. D., and Thomas, J. O. (1974). Chromatin structure; oligomers of the histones. Science 184, 865-868. doi: 10.1126/science.184.4139.865

Kumar, S., Cheng, X., Klimasauskas, S., Mi, S., Posfai, J., Roberts, R. J., et al. (1994). The DNA (cytosine-5) methyltransferases. Nucleic Acids Res. 22, 1-10. doi: 10.1093/nar/22.1.1

Kunert, N., Marhold, J., Stanke, J., Stach, D., and Lyko, F. (2003). A Dnmt2-like protein mediates DNA methylation in Drosophila. Development 130, 5083-5090. doi: $10.1242 /$ dev.00716

Lancelot, J., Caby, S., Dubois-Abdesselem, F., Vanderstraete, M., Trolet, J., OliveiraG., et al. (2013). Schistosoma mansoni Sirtuins: characterization and 
potential as chemotherapeutic targets. PLoS Negl. Trop. Dis. 7:e2428. doi: 10.1371/journal.pntd.0002428

Lepesant, J. M., Cosseau, C., Boissier, J., Freitag, M., Portela, J., Climent, D., et al. (2012). Chromatin structural changes around satellite repeats on the female sex chromosome in Schistosoma mansoni and their possible role in sex chromosome emergence. Genome Biol. 13, R14. doi: 10.1186/gb-2012-13-2-r14

Lepesant, J. M., Grunau, C., and Cosseau, C. (2011). Towards an understanding of the epigenetics of schistosomes: a comparative epigenomic study. Mem. Inst. Oswaldo Cruz. 106, 823-830. doi: 10.1590/S0074-02762012000100022

Li, B., Carey, M., and Workman, J. L. (2007). The role of chromatin during transcription. Cell 128, 707-719. doi: 10.1016/j.cell.2007.01.015

Luger, K., Dechassa, M. L., and Tremethick, D. J. (2012). New insights into nucleosome and chromatin structure: an ordered state or a disordered affair? Nat. Rev. Mol. Cell Biol. 13, 436-447. doi: 10.1038/nrm3382

Luger, K., Mäder, A. W., Richmond, R. K., Sargent, D. F., and Richmond, T. J. (1997). Crystal structure of the nucleosome core particle at 2.8 A resolution. Nature 389, 251-260. doi: 10.1038/38444

Lyko, F., Ramsahoye, B. H., and Jaenisch, R. (2000). DNA methylation in Drosophila melanogaster. Nature 408, 538-540. doi: 10.1038/35046205

Malmquist, N. A., Moss, T. A., Mecheri, S., Scherf, A., and Fuchter, M. J. (2012). Small-molecule histone methyltransferase inhibitors display rapid antimalarial activity against all blood stage forms in Plasmodium falciparum. Proc. Natl. Acad. Sci. U.S.A. 109, 16708-16713. doi: 10.1073/pnas. 1205414109

Marek, M., Kannan, S., Hauser, A. T., Moraes Mourão, M., Caby, S., Cura, V., et al. (2013). Structural basis for the inhibition of histone deacetylase 8 (HDAC8), a key epigenetic player in the blood fluke Schistosoma mansoni. PLoS Pathog. 9:e1003645. doi: 10.1371/journal.ppat.1003645

Mariño-Ramírez, L., Jordan, I. K., and Landsman, D. (2006). Multiple independent evolutionary solutions to core histone gene regulation. Genome Biol. 7:R122. doi: 10.1186/gb-2006-7-12-r122

Melters, D. P., Paliulis, L. V., Korf, I. F., and Chan, S. W. (2012). Holocentric chromosomes: convergent evolution, meiotic adaptations, and genomic analysis. Chromosome Res. 20, 579-593. doi: 10.1007/s10577-012-9292-1

Mourão, M. M., Grunau, C., LoVerde, P. T., Jones, M. K., and Oliveira, G. (2012). Recent advances in Schistosoma genomics. Parasite Immunol. 34, 151-162. doi: 10.1111/j.1365-3024.2011.01349.x

Niu, W., Lu, Z. J., Zhong, M., Sarov, M., Murray, J. I., Brdlik, C. M., et al. (2011). Diverse transcription factor binding features revealed by genome-wide ChIPseq in C. elegans. Genome Res. 21, 245-254. doi: 10.1101/gr.114587.110

Perrin, C., Lepesant, J. M., Roger, E., Duval, D., Fneich, S., Thuillier, V., et al. (2013). Schistosoma mansoni mucin gene (SmPoMuc) expression: epigenetic control to shape adaptation to a new host. PLoS Pathog. 9:e1003571. doi: 10.1371/journal.ppat.1003571

Pierce, R. J., Dubois-Abdesselem, F., Lancelot, J., Andrade, L., and Oliveira, G. (2012). Targeting schistosome histone modifying enzymes for drug development. Curr. Pharm. Des. 18, 3567-3578. doi: 10.2174/138161212801327248

Rando, O. J. (2011). Genome-wide measurement of histone H3 replacement dynamics in yeast. Methods Mol. Biol. 759, 41-60. doi: 10.1007/978-1-61779173-4_3

Rosso, M. N., Favery, B., Piotte, C., Arthaud, L., De Boer, J. M., Hussey, R. S., et al. (1999). Isolation of a cDNA encoding a beta-1,4-endoglucanase in the root-knot nematode Meloidogyne incognita and expression analysis during plant parasitism. Mol. Plant-Microbe Interact. 12, 585-591. doi: 10.1094/MPMI.1999.12.7.585

Semblat, J. P., Bongiovanni, M., Wajnberg, E., Dalmasso, A., Abad, P., and Castagnone-Sereno, P. (2000). Virulence and molecular diversity of parthenogenetic root-knot nematodes, Meloidogyne spp. Heredity 84, 81-89. doi: 10.1046/j.1365-2540.2000.00633.x
Simpson, V. J., Johnson, T. E., and Hammen, R. F. (1986). Caenorhabditis elegans DNA does not contain 5-methylcytosine at any time during development or aging. Nucleic Acids Res. 14, 6711-6719. doi: 10.1093/nar/14.16.6711

Strahl, B. D., and Allis, C. D. (2000). The language of covalent histone modifications. Nature 403, 41-45. doi: 10.1038/47412

Subirana, J. A., and Messeguer, X. (2013). A satellite explosion in the genome of holocentric nematodes. PLoS ONE 8:e62221. doi: 10.1371/journal.pone.0062221

Suzuki, M. M., and Bird, A. (2008). DNA methylation landscapes: provocative insights from epigenomics. Nat. Rev. Genet. 9, 465-476. doi: 10.1038/nrg2341

Triantaphyllou, A. C. (1981). Oogenesis and the Chromosomes of the Parthenogenic Root-knot Nematode Meloidogyne incognita. J. Nematol. 13, 95-104.

Triantaphyllou, A. C. (1983). “Cytogenetic aspects of nematode evolution," in Concepts in Nematode Systematics, eds A. R. Stone, H. M. Platt, and L. F. Khali (London: Academic Press), 55-71.

Trojer, P., and Reinberg, D. (2007). Facultative heterochromatin: is there a distinctive molecular signature? Mol. Cell 28, 1-13. doi: 10.1016/j.molcel.2007. 09.011

Turner, B. M. (1993). Decoding the nucleosome. Cell 75, 5-8. doi: 10.1016/00928674(93)90673-E

Turner, B. M. (2005). Reading signals on the nucleosome with a new nomenclature for modified histones. Nat. Struct. Mol. Biol. 12, 110-112. doi: 10.1038/nsmb0205-110

Turner, B. M. (2012). The adjustable nucleosome: an epigenetic signaling module. Trends Genet. 28, 436-444. doi: 10.1016/j.tig.2012.04.003

Urieli-Shoval, S., Gruenbaum, Y., Sedat, J., and Razin, A. (1982). The absence of detectable methylated bases in Drosophila melanogaster DNA. FEBS Lett. 146, 148-152. doi: 10.1016/0014-5793(82)80723-0

Verhoeven, K. J., and Preite, V. (2014). Epigenetic variation in asexually reproducing organisms. Evolution 68, 644-655. doi: 10.1111/evo.12320

Wenzel, D., Palladino, F., and Jedrusik-Bode, M. (2011). Epigenetics in C. elegans: facts and challenges. Genesis 49, 647-661. doi: 10.1002/dvg.20762

Winnepenninckx, B., Backeljau, T., and De Wachter, R. (1993). Extraction of high molecular weight DNA from molluscs. Trends Genet. 9, 407. doi: 10.1016/01689525(93)90102-N

Youngson, N. A., and Whitelaw, E. (2008). Transgenerational epigenetic effects. Annu. Rev. Genomics Hum. Genet. 9, 233-257. doi: 10.1146/annurev.genom.9.081307.164445

Conflict of Interest Statement: The authors declare that the research was conducted in the absence of any commercial or financial relationships that could be construed as a potential conflict of interest.

Received: 09 December 2013; accepted: 15 May 2014; published online: 06 June 2014. Citation: Perfus-Barbeoch L, Castagnone-Sereno P, Reichelt M, Fneich S, Roquis D, Pratx L, Cosseau C, Grunau C and Abad P (2014) Elucidating the molecular bases of epigenetic inheritance in non-model invertebrates: the case of the root-knot nematode Meloidogyne incognita. Front. Physiol. 5:211. doi: 10.3389/fphys.2014.00211

This article was submitted to Invertebrate Physiology, a section of the journal Frontiers in Physiology.

Copyright (c) 2014 Perfus-Barbeoch, Castagnone-Sereno, Reichelt, Fneich, Roquis, Pratx, Cosseau, Grunau and Abad. This is an open-access article distributed under the terms of the Creative Commons Attribution License (CC BY). The use, distribution or reproduction in other forums is permitted, provided the original author(s) or licensor are credited and that the original publication in this journal is cited, in accordance with accepted academic practice. No use, distribution or reproduction is permitted which does not comply with these terms. 